\title{
The ocular protective effects of PLGA nanoparticles loaded with lutein, in fat diet Wistar rats, treated with sistemic glucocorticoids
}

\author{
Adriana DINU, Bogdana VIRGOLICI, Daniela MIRICESCU, Alexandra TOTAN, Horia VIRGOLICI, \\ Maria GREABU, Maria MOHORA \\ "Carol Davila" University of Medicine and Pharmacy, Bucharest, Romania
}

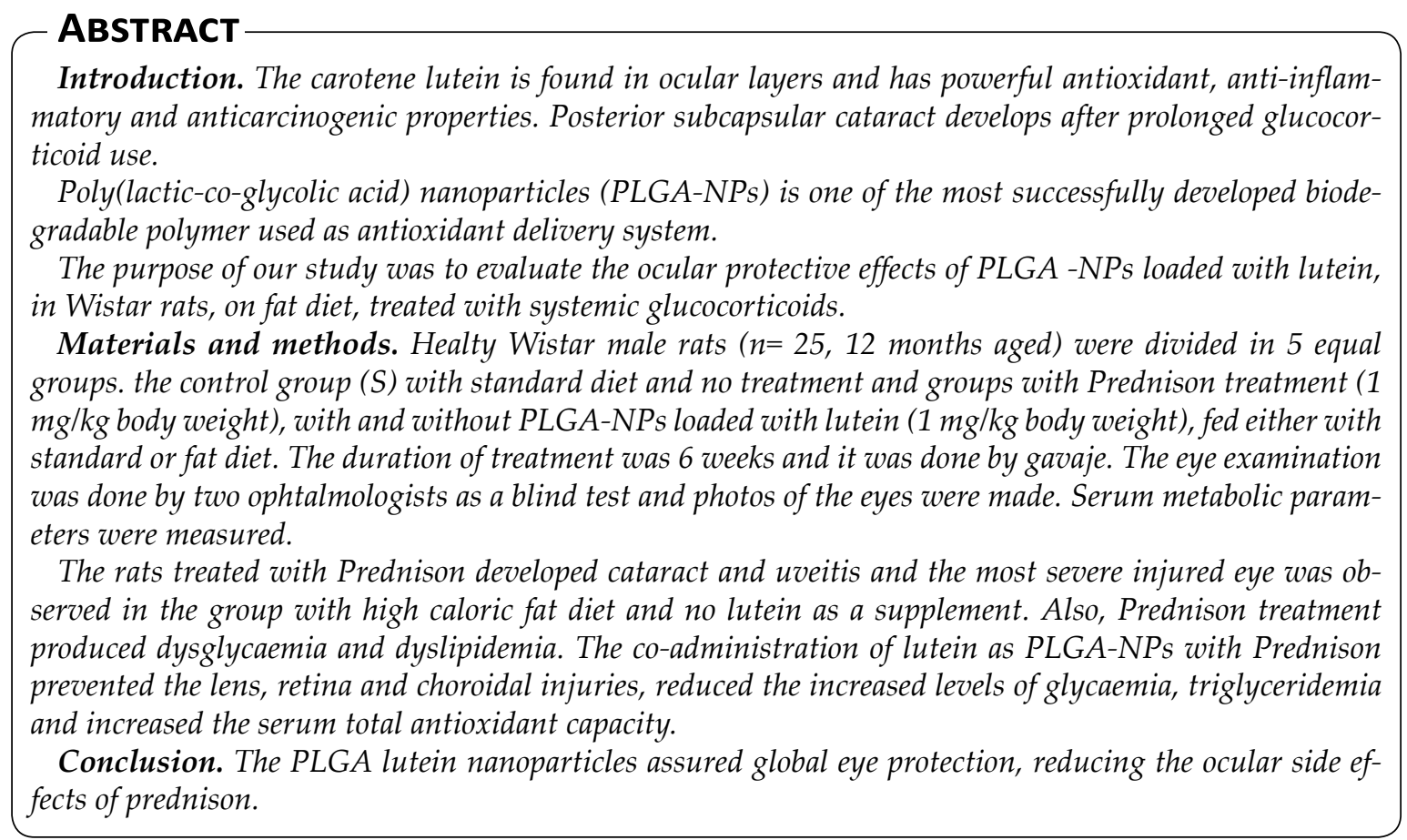

Keywords: lutein PLGA-Ns, ocular protection, glucocorticoids, Wistar rats

\section{INTRODUCTION}

Glucocorticoids are important immunosuppressive and anti-inflammatory drugs.They are widely used in the treatment of autoimmune disorders, and other conditions such as asthma, skin disorders, allergic reactions. The anti-inflammatory and immunosuppressive effects are manifested 
by reducing the expression of pro-inflammatory cytokines, adhesion molecules, circulating monocytes, macrophages [1].

Long-term administration of glucocorticoids such as prednisone has side effects and is correlated with the occurrence of fractures, infections and cataracts [2].

Glucocorticoids have metabolic side effects, increase the risk of hyperglycemia, in a dose- and time-dependent manner in healthy and diabetic patients. Hyperglycemia may appear at a daily prednisone concentration of $2.5 \mathrm{mg} /$ day [2].

Worldwide, cataracts are a major cause of blindness and vision impairment among the adult population.Cataracts usually develop over the age of 50 , with a higher rate in sunny areas and higher incidence in women than in men [3].

Nowadays, surgery is the only option for cataract patients for vision recovery [4]. Lutein is a carotenoid with anti-inflammatory properties that present beneficial effects on eye health. Lutein administration improves or even prevents age- related macular degeneration and cataract. Food lutein is absorbed by small intestine mucosa, embedded in chylomicrons, and transported to the liver. From the liver to the peripheral tissues, such as the retina, lutein is loaded in chylomicrons structure. Lutein absorption can be affected by protein, Fe and $\mathrm{Zn}$ deficiency [5].

PLGA (poli-lactic-CO-glycolic acid) nanoparticles loaded with lutein is an excellent alternative to remove these inconvenience. PLGA is a biodegradable polymer, whose hydrolytic degradation leads to lactic and glycolic acid, metabolized via the Krebs cycle, with minimal side effects. Pinocytosis and claritine-mediated endocytosis are the mechanisms of PLGA internalization in cells.PLGA reaches the cytoplasm in less than 10 minutes after incubation, rapidly escaping endo-lysosomes [6].

The aim of our study was to test the antioxidant effects of PLGA-loaded with lutein in Wistar rats with cataracts induced by prednisone administration and hypercaloric diet.

\section{MATERIALS \& METHODS}

Old Wistar male rats ( 12 months old, $\mathrm{n}=25$, weight $=380-410 \mathrm{~g})$ raised on standard $\operatorname{diet}(3.5$ $\mathrm{kcal} / \mathrm{g})$, were randomly divided into five experimental groups ( $n=5$ for each group).Then for 6 weeks, the experimental animals were fed with different diet. Group S (control) and Group SP (standard-prednison) continued this diet without or with Prednisone $(1 \mathrm{mg} / \mathrm{kg} /$ day). Group F (fat) and FP (fat- prednisone) were fed with a high ca- loric/high fat diet $(5.15 \mathrm{kcal} / \mathrm{g})$ without or with Prednisone. FPL group (fat- prednisone- lutein) received high fat diet and treatment with Prednisone and Lutein PLGA-NPs ( $1 \mathrm{mg} / \mathrm{kg}$ body)in the same time. The treatment was done by oral gavage. The rats were hold in cages at $20-22^{\circ} \mathrm{C}$ temperature, $12 \mathrm{~h}$ light and $12 \mathrm{~h}$ dark cycles, humidity $50 \pm 5 \%$ and had access to standard rodent meal and water ad libitum.

Lutein PLGA-NPswereobtained from the Department of Biological and Agricultural Engineering, Louisiana State University, 70803, Louisiana, United States and Prednisone (Prednisone-Richter, $5 \mathrm{mg} /$ tablet) was from Gedeon Richter Romania S.A).

PLGA polymeric nanoparticles, $95 \pm 2 \mathrm{~nm}$ were spherical in shape and had a negative zeta potential $(-38 \mathrm{mV})$. Before being sacrificed the animals were fasted for $12 \mathrm{~h}$ and sedated with Ketamine (25 mg/kg weight). Blood samples and the eyes were collected from each animal.

\section{Biochemical analysis}

Blood samples were centrifuged at 3,000 rpm for $5 \mathrm{~min}$ and the serum was used for biological measurements. Kits had been provided from Biosystems (Spain). It was respected manufacturer's indications.

Total antioxidant capacity assay (TAC) sau (Total Antioxidant Status-TAS, Randox, Crumlin, UK activity was performed using an analysis kit), on a semiautomatic biochemistry analyzer. The method is based on the ability of antioxidant molecules to quench the long-lived ABTSæ\%+, a bluegreen chromophore with characteristic absorption at $734 \mathrm{~nm}$, in comparison to that of Trolox, a water-soluble vitamin $\mathrm{E}$ analogue.

Statistical analysis was performed using a Student's t-test (two tailed, unpaired). Mean \pm standard deviation (SD) values was calculated. Statistical significance was set at a p-value of < 0.05 .

The EU Directive 2010/63 approved in 2014, was respected regarding the protection of animals used for experimental and other scientific purposes.The experiment was accomplished at the Biobase of the Faculty of Medicine, Carol Davila University of Medicine and Pharmacy, Bucharest, Romania.

\section{RESULTS}

The serum biological results and clinical examination results are depicted in the following tables and figures. 


\section{DISCUSSION}

The main cause of blindness in the world is cataract and $50 \%$ of the populations over 80 have loss of vision because of cataract. Light and oxygen are environmental agents that produce free oxygen species and eye is a sensitive organ to oxidative stress. Cataract, glaucoma and inflammation are common diseases of the human eye and antioxidant supplements have beneficial effects [7-9].

In animal models, for cataract screening, researchers have been used in vivo and in vitro (incubated lenses) experiments with triggers like: sel-

TABLE 1. Comparison of the average values of serum parameters for groups: $S$ and $F$

\begin{tabular}{|l|c|c|c|}
\hline \multicolumn{1}{|c|}{ Parameters } & S group & F group & F vs S \\
\hline Cortisol $\mu \mathrm{g} / \mathrm{dl}$ & $7.25 \pm 1,06$ & $9.45 \pm 2.33$ & $\mathrm{Ns}$ \\
\hline FBG $\mathrm{mg} / \mathrm{dl}$ & $86 \pm 2.62$ & $109.5 \pm 4.94$ & $\mathrm{p}<0.0001$ \\
\hline $\mathrm{Hb} \mathrm{A1c \%}$ & $3.8 \pm 0.02$ & $4.9 \pm 0.14$ & $\mathrm{p}<0.0005$ \\
\hline lonic Na mmol/l & $124.56 \pm 2.85$ & $195.23 \pm 8.26$ & $\mathrm{Ns}$ \\
\hline Triglycerides $\mathrm{mg} / \mathrm{dl}$ & $96.5 \pm 4.94$ & $241.5 \pm 12.79$ & $\mathrm{p}<0.0035$ \\
\hline HDL cholesterol mg/dl & $24 \pm 4.24$ & $23 \pm 2.82$ & $\mathrm{Ns}$ \\
\hline Cholesterol mg/dl & $88.5 \pm 12.02$ & $76.2 \pm 13.43$ & $\mathrm{Ns}$ \\
\hline TAC mmol/l & $5.25 \pm 0.49$ & $4.25 \pm 1.9$ & $\mathrm{Ns}$ \\
\hline
\end{tabular}

FBG fasting blood glucose, Hb A1c glycated hemoglobin, Na serum sodium HDL high density lipoprotein TAC total antioxidant capacity

TABLE 2. Comparison of the average values of serum parameters for groups: $S$ and $S P$

\begin{tabular}{|l|c|c|c|}
\hline \multicolumn{1}{|c|}{ Parameters } & S group & SP group & S vs SP \\
\hline Cortisol $\mu \mathrm{g} / \mathrm{dl}$ & $7.25 \pm 1,06$ & $9.2 \pm 3.02$ & $\mathrm{p}<0.001$ \\
\hline FBG $\mathrm{mg} / \mathrm{dl}$ & $86 \pm 2.62$ & $106.75 \pm 3.59$ & $\mathrm{p}<0.0001$ \\
\hline $\mathrm{Hb} \mathrm{A} 1 \mathrm{C} \%$ & $3.8 \pm 0.02$ & $5.05 \pm 0.91$ & $\mathrm{p}<0.02$ \\
\hline Ionic Na mmol/l & $124.56 \pm 2.85$ & $132.7 \pm 6.08$ & $\mathrm{p}<0.026$ \\
\hline Triglycerides mg/dl & $96.5 \pm 4.94$ & $134.8 \pm 21.85$ & $\mathrm{p}<0.005$ \\
\hline HDL cholesterol mg/dl & $24 \pm 4.24$ & $20.04 \pm 3.4$ & $\mathrm{p}<0.05$ \\
\hline Cholesterol mg/dl & $88.5 \pm 12.02$ & $118.8 \pm 13.55$ & $\mathrm{p}<0.0006$ \\
\hline TAC $\mathrm{mmol} / \mathrm{l}$ & $5.25 \pm 0.49$ & $4.775 \pm 0.63$ & $\mathrm{Ns}$ \\
\hline
\end{tabular}

FBG fasting blood glucose, Hb A1c glycated hemoglobin, Na serum sodium HDL high density lipoprotein TAC total antioxidant capacity enite, naphthalene, $\mathrm{H}_{2} \mathrm{O}_{2}$, etc [10]. In the present experimental study, Prednison was given by gavaje, for 6 weeks, in Wistar rats with two types of diet, standard and obesogenic.

Many types of cataract: nuclear, cortical and posterior subcapsular cataract have been described [11]. Corticosteroids increase the risk of posterior subcapsular type of cataract. It seems that steroids do not directly act on the lens but rather produce an imbalance of ocular cytokines and growth factors [12]. Recently, it was demonstrated that distinct metabolic compartments exist in the lens and these explains different etiologies of nuclear and cortical cataract, even the one induced by Prednison [13].

Dysglycaemia and oxidative stress are the principal culprits in almost all types of cataract [14]. Lower levels of systemic and lens antioxidants including lutein and reduced glutathione (GSH) are associated with cataract [15].

In order to restore the depleted antioxidant level in the lens, it is important to enhance the delivery and accumulation of antioxidants in the lens. In Sprague-Dawley rats with Dexamethasone $(5 \mu \mathrm{M})$ induced cataract, $\mathrm{N}$-acetylcysteine amide elevated the GSH/GSSG ratio in the lenses, limiting the lipid peroxidation [16]. In other experimental studies, the benefic effect of carotenoids was explained by increasing the antioxidant defence in the eyes, but, interesting, only in $\mathrm{H} 2 \mathrm{O} 2$ induced cataract, lutein didn't mitigate the low level of GSH [10].

Lutein is a beta-carotene which accumulates preferentially in the macula lutea. It defenses oxidative damage from ultraviolet light and it is known as a protective factor in age macular degeneration [5]. But, lutein, also delays the cataract onset and progression. For these reason it can be recommended as a supplement for eye diseases $[17,18]$.

TABLE 3. Comparison of the average values of serum parameters for groups: F and PF and also for PFL and PF

\begin{tabular}{|l|c|c|c|c|c|}
\hline Parameters & F group & PF group & F vs PF & PFL group & PF vs PFL \\
\hline Cortisol $\mu \mathrm{g} / \mathrm{dl}$ & $9.45 \pm 2.33$ & $6.1 \pm 1.13$ & $\mathrm{p}<0.02$ & $7.92 \pm 3.22$ & $\mathrm{Ns}$ \\
\hline FBG $\mathrm{mg} / \mathrm{dl}$ & $109.5 \pm 4.94$ & $121 \pm 7.07$ & $\mathrm{p}<0.01$ & $96 \pm 18.16$ & $\mathrm{p}^{\prime}<0.02$ \\
\hline $\mathrm{Hb} \mathrm{A1C} \%$ & $4.9 \pm 0.14$ & $5.9 \pm 0.42$ & $\mathrm{p}<0.001$ & $4.775 \pm 0.74$ & $\mathrm{p}^{\prime}<0.01$ \\
\hline lonic Na mmol/l & $195.23 \pm 8.26$ & $136 \pm 22.7$ & $\mathrm{Ns}$ & $132.09 \pm 15.8$ & $\mathrm{Ns}$ \\
\hline Triglycerides $\mathrm{mg} / \mathrm{dl}$ & $241.5 \pm 12.79$ & $403.5 \pm 30.4$ & $\mathrm{p}<0.001$ & $342 \pm 49.5$ & $\mathrm{p}^{\prime}<0.02$ \\
\hline HDL cholesterol mg/dl & $23 \pm 2.82$ & $17.7 \pm 2.5$ & $\mathrm{p}<0.05$ & $19.8 \pm 2.8$ & $\mathrm{Ns}$ \\
\hline Cholesterol mg/dL & $76.2 \pm 13.43$ & $91.5 \pm 12.02$ & $\mathrm{p}<0.026$ & $66.5 \pm 12.81$ & $\mathrm{p}^{\prime}<0.013$ \\
\hline TAC $\mathrm{mmol} / \mathrm{l}$ & $4.25 \pm 1.9$ & $3.1 \pm 1.31$ & $\mathrm{Ns}$ & $5.1 \pm 0.99$ & $\mathrm{p}^{\prime}<0.017$ \\
\hline
\end{tabular}

FBG fasting blood glucose, Hb A1c glycated hemoglobin, Na serum sodium

HDL high density lipoprotein TAC total antioxidant capacity 
TABLE 4. Clinical eye examination results

\begin{tabular}{|c|c|c|}
\hline Wistar group & Clinical examination 1 & Clinical examination 2 \\
\hline S group-transparent lens & & \\
\hline $\begin{array}{l}\text { SP group-cataract, uveitis and } \\
\text { rubeosis iridis }\end{array}$ & & \\
\hline F group-cataract and uveitis & & \\
\hline $\begin{array}{l}\text { PF group-glaucoma, cataract } \\
\text { rubeosis iridis }\end{array}$ & & \\
\hline PFL group almost normal aspect & & \\
\hline
\end{tabular}

Metabolic disorders (obesity, diabetes mellitus), life style (long use of steroids, smoking), aging and poor diet are important risk factors for cataract.

In our study, the rats on obesogenic diet, associated with Prednison had the "worst picture" of metabolic parameters and eye aspect. The high caloric/high fat diet increased the levels of fasting plasma glucose, of glycated hemoglobin and of serum triglycerides and didn't influence significantly the levels of total antioxidant capacity and cholesterolemia (Table 1). Each time, when rats where treated with Prednison, regardless the diet (obesogenic or standard), dysglycaemia and dyslipidemia were present (Table 2). Our results are in accordance with clinical studies which demonstrated that there is a negative correlation between cortisol and cholesterol/HDLc ratio and a positive correlation between saliva cortisol and fasting glycaemia and between Dexamethasone treatment and high glycaemia [19-21].

By MRI imaging, it was demonstrated that the lens has a unique internal microcirculation system to deliver antioxidants in different regions, and sodium is important in this mechanism [13]. In both groups treated only with Prednison, the serum level of ionic sodium was significantly increase (Tables 2 and 3). Maybe serum sodium can influence the ocular level of this metal.

In the high fat diet Wistar rats, the association of lutein loaded PLGA-NPs to Prednison, decreased 
the increased values for glycaemia, triglyceridemia and improved the systemic antioxidant defence of the body. Bodoki et al., by comparison free lutein or lutein-loaded NPs administered orally or topically in selenite cataract, demonstrated that the severity of lens injury was significantly reduced in rats treated with topical applications of lutein-loaded NPs [22]. Recently, it was demonstrated that in diabetic cataract, autoregenerative redox nanoparticles can act as an antioxidant and glycation inhibitor [23].

Experimental studies demonstrated that lutein has also hepatoprotective effects, "fighting "against xenobiotics and reversing the histopathological abnormalities. This antioxidant reduced the elevated levels of ALT and AST enzymes in ethanol treated rats [24]. Also, it was demonstrated that PLGA-NPs loaded with vitamin $E$ had visceral tissue protective effects and lowered cholesterolemia [25].

In accordance with the dysmetabolic modifications, oral treatment with glucocorticoids produced eye injuries: cataract, uveitis, glaucoma and rubeosis iridis. While cataract is the clouding of the lens, the rubeosis iridis is associated with impaired ciliary body perfusion, with a subsequent decrease in aqueous production [26].

The eyes from $\mathrm{S}$ group, control group, healthy group, on standard diet had transparent corneea and crystallin, while the eyes from SP Group, with prednison treatment $(1 \mathrm{mg} / \mathrm{kg}$ body weight), standard diet had uveitis and rubeosis iridis (Table 4).

In the eyes from $\mathrm{F}$ group with fat diet, cataract and uveitis were observed.

In group $\mathrm{PF}$, with prednison treatment $(1 \mathrm{mg} /$ kg body weight) and fat diet, glaucoma, cataract and rubeosis iridis were present (Table 4).

Group PFL with Prednison treatment $(1 \mathrm{mg} / \mathrm{kg}$ body weight), fat diet and PLGA NPs loaded with lutein ( $1 \mathrm{mg} / \mathrm{kg}$ body weight), normal aspect is observed. The association of lutein to the glucocorticoids prevented the inflammatory and degenerative modifications in the eye (Table 4).

\section{CONCLUSION}

PLGA-NPs loaded with lutein (1 $\mathrm{mg} / \mathrm{kg}$ body) had ocular protective effects in high fat diet Wistar rats treated with Prednison ( $1 \mathrm{mg} / \mathrm{kg}$ body) and improved sugar and lipid metabolism.

\section{Acknowledgments}

Authors thank to Assoc. Prof. Iuliana Ionascu, $\mathrm{MD}, \mathrm{PhD}$, University of Agronomic Sciences and Veterinary Medicine, 59 Mărăşti Blvd., 011464, Bucharest, Romania) and to lecturer Ramona Barac, MD, PhD, Department of Ophtalmology, Carol Davila University of Medicine and Pharmacy, 8 Eroilor Sanitari, 050474, Bucharest, Romania.

All the authors had equal contribution.

Conflict of interest: none declared Financial support: none declared

\section{REFERENCES}

1. Buttgereit F, Saag KG, Cutolo M et al. The molecular basis for the effectiveness, toxicity and resistance to glucocorticoids: Focus on the treatment of rheumatoid arthritis. Scand J Rheumatol 2005;34:1421.

2. Clore JN, Thurby-Hay L. Glucocorticoidinduced hyperglycemia. Endocr Pract 2009;15:469-74.

3. Lee CM, Afshari NA. The global state of cataract blindness. Curr Opin Ophthalmol 2017;28(1):98-103.

4. Prokofyeva E, Wegener A, Zrenner E. Cataract prevalence and prevention in Europe: A literature review. Acta Ophthalmol 2013;91(5):395-405.

5. Buscemi S, Corleo D, Di Pace F et al. The Effect of Lutein on Eye and Extra-Eye Health. Nutrients 2018; 10:1321.

6. Danhier F, Ansorena E, Silva JM et al. PLGA-based nanoparticles: An overview of medical applications. J Control Release 2012;161(2):505-22.
7. Gopal PNV, Shaik SS, Grandhi S et al. Phytochemical Review on Cataract. World Journal of Pharmaceutical Sciences 2016; ISSN (Print): 2321-3310; ISSN (Online): 2321-3086,

8. Babizhayev MA, Wan-Cheng LiD, Kasus-Jacobi A et al. Studies on the Corneea and Lens. Springer - Humana Press 2015.

9. Wong L, Ho SC, Coggon, D et al. Sunlight exposure, antioxidant status, and cataract in Hong Kong fishermen. J Epidemiol and Community Health 1993; 47:46-49.

10. Heruye SH, Maffofou Nkenyi, LN et al. Current Trends in the Pharmacotherapy of Cataracts. Pharmaceuticals (Basel) 2020; 16;13(1).

11. West SK, RosenthalF, Newlnd HS et al. Use of photographic tehniques tograde nuclear cataract. Investig Ophtamol Vis Science 1988; 29:73-77.

12. Jobling Al, Augusteyn RC, What causes steroid cataracts? A review of steroid- induced posterior subcapsular cataracts. Clin Exp Optom 2002; 85:2: 61-75.

13. Braakhius AJ, Donaldson Cl, Lim J C et al. Nutritional Strategies to prevent lens cataract: current status and future strategies. Nutrients 2019; 11(1186):10003.

14. Hedge KR, Varma SD, Synergistic effect of glycemic and oxidative stress in cataract formation. Invest Ophthalmol Vis Sci 2004;45: E-Abstract 1706.

15. Jacques PF, Chylack Jr. LT, McGandy RB et al. Antioxidant status in persons with and without senile cataract. Arch Ophthalmol 1988;106:337-40.

16. Tobwala S, Pinarci EY, Maddirala Yet al. $\mathrm{N}$-acetylcysteine amide protects against dexamethasone-induced cataract related changes in cultured rat lenses. Adv Biol Chem 2014; 4: 26-34.

17. Manayi A, Abdollahi M, Raman T et al. Lutein and cataract: from bench to bedside. Crit Rev Biotechnol 2016;36(5):829-39. 
18. Chew EY, SanGiovanni JP, Ferris FL et al. Age-Related Eye Disease Study 2 (AREDS2) Research Group. JAMA Ophthalmol 2013;131(7):843-50.

19. Maduka IC, Neboh E, Ufelle SA. The relationship between serum cortisol, adrenaline, blood glucose and lipid profile of undergraduate students under examination stress. African Health Sciences 2015; 15(1):131-136

20. Deneva T, lanakiev Y, Keskinova D. Burnout Syndrome in PhysiciansPsychological Assessment and Biomarker Research. Medicina (Kaunas) 2019;24;55(5)
21. Papacocea T, Popa E, Turliuc D et al. Theesefulness of Dexamethasone in the treatment of chronic subdural hematomas. Farmacia, 2019;67(1):140-145.

22. Bodoki E, Vostinaru O, Samoila O et al. Topical nanodelivery sistem of lutein fer the prevention of selenite-induced cataract. Nanomedicine, Nanotechnology, Biology, and Medicine 2019; 15:188-197.

23. ZhouY, Li L, Shenghui Li et al. Autoregenerative redox nanoparticles as an antioxidant and glycation inhibitor for palliation of diabetic cataracts. Nanoscale 2019; 27.
24. Faria EC, Ambrosano AML, Lage R et al. Nutraceuticals for Healthy Skin Aging. Chapter 23, Nutrition and Functional Foods for Healthy Aging 2017;273-281.

25. Balaban M, Vîrgolici B, Dinu A et al. Metabolic Parameters in Wistar Rats Treated with Glucocorticosteroids and Vitamin E-Charged Poly Lactic-Co-Glycolic Acid (PLGA) Nanoparticles. Rev Chim (Bucharest) 2019; 70( 4):1315-1318.

26. Sharma S, Brown GC. Chapter 84 - Ocular Ischemic Syndrome, Retina (Fourth Edition) 2006; 2:1491-1502. 